\title{
Motives to Drink as Mediators Between Childhood Sexual Assault and Alcohol Problems in Adult Women
}

\author{
Carla E. Grayson ${ }^{1,2}$ and Susan Nolen-Hoeksema ${ }^{1}$
}

\begin{abstract}
Two models are proposed to relate maladaptive emotion regulation strategies and alcohol-related problems for women with a history of childhood sexual assault (CSA). The distress coping model suggests only one motive-drinking to cope with negative emotions-mediates the relationship between CSA and alcohol problems. The emotion regulation model suggests two motives mediate the relationship between CSA and alcohol problems: drinking to cope with negative emotions and drinking to enhance positive emotions. These models were tested in a random community sample of 697 women, ranging from 25 to 75 years old. Both motives partially mediated the relationship between CSA and alcohol problems. Effects were small, but reliable.
\end{abstract}

The experience of childhood sexual assault (CSA) is associated with a number of long-term adverse consequences (for reviews, see Davis \& Petretic-Jackson, 2000; Polusny \& Follette, 1995). Women who have experienced CSA have high rates of depression (e.g., Weiss, Longhurst, \& Mazure, 1999; Wilsnack, Vogeltanz, Klassen, \& Harris, 1997), anxiety (Jarvis \& Copeland, 1997; Neumann, Houskamp, Pollock, \& Briere, 1996), and posttraumatic stress disorder (Saunders, Villeponteaux, Lipovsky, Kilpatrick, \& Veronen, 1992). Other long-term correlates of CSA include maladaptive behaviors such as heavy drinking (for reviews, see Langeland \& Hartgers, 1998; Moncrieff \& Farmer, 1998), high-risk sexual behavior (Wyatt, 1988; Zierler et al., 1991), and disordered eating (e.g., Romans, Gendall, Martin, \& Mullen, 2001).

In a review of the long-term correlates of CSA, Polusny and Follette (1995) outlined a conceptual model that could explain the relationship between CSA and a number of maladaptive behaviors including excessive al-

\footnotetext{
${ }^{1}$ Department of Psychology, Institute for Research on Women and Gender, University of Michigan, Ann Arbor, Michigan.

${ }^{2}$ To whom correspondence should be addressed at Department of Psychology, University of Michigan, 530 Church Street, Ann Arbor, Michigan 48109-1043; e-mail: carlag@umich.edu.
}

cohol consumption. They proposed that people with a history of CSA will engage in behaviors, such as drinking, that will allow them to cope with their chronic distress (see also Hayes, 1987). In fact, female survivors of childhood sexual assault report more problems with alcohol than controls (Moncrieff \& Farmer, 1998). According to this model, women with a CSA history would drink primarily to cope with their negative internal psychological states. This idea is consistent with more general models of drinking behavior. For example, tension reduction theory (TRT; Conger, 1956) predicts that people will drink to decrease negative affect. Empirical evidence has generally supported the proposed relationship between stressors and alcohol consumption (for a review, see Pohorecky, 1991).

Childhood sexual assault may be associated with more generalized emotion-regulation deficits than a limited ability to cope with distress. Learning to achieve positive affective states, such as contentment, satisfaction, and happiness also is an important developmental task (Vaillant, 2000). To the degree that CSA interferes with development of adaptive emotion-coping strategies, it is plausible functional skills in creating positive affect also are influenced. Cooper and colleagues (Cooper, Agocha, \& Sheldon, 2000; Cooper, Frone, Russell, \& Mudar, 1995; Cooper, Russell, Skinner, \& Windle, 1992) proposed a 
general model of motives in which people engage in risky activities to manage both their negative and positive emotions. For example, individuals may drink to cope with their distress or to achieve or enhance a positive mood. In a study of adolescents with histories of both physical and sexual assault, Harrison, Fulkerson, and Beebe (1997) found female assault victims were more than twice as likely to use substances to reduce painful emotions than nonvictims and over 1.5 times more to escape from problems. Victims also were over 1.5 times more likely to drink to get high and to relax than nonvictims. In addition, CSA has been linked to the use of stimulants such as amphetamines and cocaine (Jarvis, Copeland, \& Walton, 1998), suggesting CSA may lead to maladaptive strategies for seeking positive affect.

While the coping and enhancement motives are related, they are conceptually distinct. A considerable theoretical and empirical literature suggests that the experience of negative and positive emotional states are qualitatively different and not simply opposites (e.g., Diener \& Emmons, 1984). Positive affect is not simply achieved by removing negative affect. Cooper and colleagues (Cooper et al., 2000; Cooper et al., 1995) based their argument in part on evidence that positive and negative emotions have been associated with different biological systems (Gray, 1990). It is logically consistent that motivations to achieve these states can be distinguished, and empirical evidence supports this contention (Cooper et al., 1992).

The present study examined whether the emotion regulation motives mediate the relationship between CSA and problems that may result from behaviors intended to alter emotional states. More specifically, we investigated whether the relationship between CSA and alcohol problems was mediated by the coping and enhancement motives. The present study focused on alcohol problems as an outcome because we were interested in the effects of maladaptive coping mechanisms. We approached this analysis with the framework that either motive reflects an ineffective emotion regulation strategy when used chronically and may lead to problem generation rather than problem resolution (Cooper et al., 1992).

Although the relationship between CSA and alcohol problems is well documented (e.g., Wilsnack et al., 1997), there have been few empirical studies addressing the underlying psychological mechanisms that might mediate this relationship. In one of these studies, Epstein, Saunders, Kilpatrick, and Resnick (1998; but also see Nishith, Resick, \& Mueser, 2001) found that posttraumatic stress symptomatology served as a mediator between childhood rape and alcohol use in adult women. The present study extends their findings by specifying emotion regulation processes that may explain the relationship between psy- chological distress and alcohol problems. We assessed distress in this study rather than PTSD because we were interested in examining a broader range of emotional symptoms than PTSD symptoms. Additionally, Cooper et al. (1995) found that depressive symptoms predicted the coping motive. Finally, the data used for this article are from a study originally designed to examine depression and anxiety rather than PTSD. This study examined two mediational models.

The first model was based on the distress reduction framework developed by Polusny and Follette (1995). This model predicts that CSA will be associated with increased distress in adulthood. Moreover, that distress will lead to increased likelihood to drink to cope with or avoid that negative affective state. Finally, the more likely women are to drink to cope with distress, the more likely they will have alcohol-related problems. In effect, the relationship between CSA and alcohol-related problems will be mediated by distress and the coping motive. We called this model the distress coping model to indicate its focus on explaining the relationship between CSA and drinking problems using the coping motive.

We named the second model we examined the emotion regulation model to reflect its broad scope. The emotion regulation proposes that CSA is related to regulation strategies including both coping with negative emotions and seeking of positive emotions. Both motives lead women to engage in ineffective behaviors such as excessive alcohol consumption that ultimately lead to more problems rather than resolving previous ones. The emotion regulation model includes all the predictions made by the distress coping model. In addition, it adds the prediction that the motive to drink to achieve positive emotions states also will serve to mediate the relationship between CSA and alcohol problems.

\section{Method}

\section{Participants}

Participants were recruited through random-digit dialing in a major metropolitan area on the West Coast. All telephone numbers were residential. If someone in the household was between the ages of 25 and 35, 45 and 55, or 65 and 75 years old, the interviewer asked that person to participate in the study. Only one person per household was recruited. Of the 1,789 people called and identified as meeting the age criteria of the study, $19 \%$ were not interested in participating, 3\% said they did not have time to participate, and $4 \%$ initially agreed to participate but did not return repeated phone calls to schedule an interview. 
Of the 1,327 people interviewed, 697 were women. All analyses reported later in this study were conducted only on the women's data.

The participants were primarily Caucasian (71\%), with 9\% African American, 9\% Hispanic, 6\% Asian American, and 5\% other ethnicities. Thirty-seven percent of the participants were between 25 and 35 years, $40 \%$ were between 45 and 55 years, and $23 \%$ were between 65 and 75 years of age. The median income was in the range of $\$ 40,000$ to $\$ 50,000$, and median education was "some college." Twenty-four percent $(n=167)$ reported incidents of childhood sexual assault. This rate of prevalence is consistent with other community samples (e.g., Finkelhor, Hotaling, Lewis, \& Smith, 1990).

\section{Measures}

\section{Demographics}

Participant gender, age, ethnicity, educational attainment, and income were ascertained at the beginning of the interview.

\section{Childhood Sexual Assault}

Childhood sexual assault was assessed by the item "In your lifetime, has anyone ever tried to pressure or force you to have sexual contact? By sexual contact, I mean their touching your sexual parts, your touching their sexual parts, or sexual intercourse?" This item was originally developed for the Los Angeles Epidemiological Catchment Area Study and developed in collaboration with the National Institute for Mental Health (see Burnam et al., 1988). Participants who answered in the affirmative to the screening question were then asked at what age they first experienced unwanted sexual contact. For the present study, participants who reported a first incident before the age of 18 were coded as having experienced childhood sexual assault. Single-item measures are typically associated with underreporting rather than overreporting of assaults (Finkelhor \& Brown, 1988). By extension, this measure is probably a conservative estimate of sexual assault in our sample.

\section{Drinking Motives}

The enhancement and coping motives were measured using the Drinking to Enhance Positive Mood and Drinking to Cope with Distress subscales of the Drinking Motives Measure (Cooper et al., 1992). Each subscale consists of five items that tap either a tendency to drink to create a positive mood (e.g., "Because it is exciting" or "Because it makes you feel good") or to decrease distress (e.g., "To forget your worries" or "To cheer up when you're in a bad mood"). Participants were asked to rate how frequently they consumed alcohol during the last year for these motives on a 4-point scale $(1=$ "never/almost never," 2 = "sometimes," $3=$ "often," and $4=$ ="always/almost always"). Subscale scores were calculated by averaging the items. Scores ranged from 1 to 4 for drinking to cope with distress and from 1 to 3.6 for drinking to enhance positive mood. Drinking motives were set equal to 1 for participants who had not consumed alcohol in the last year. The scales had good reliability in our sample (Cronbach's $\alpha$ for Drinking to Enhance $=.83$ and for Drinking to Cope $=.81$ ). The drinking motive scales have demonstrated similar internal consistencies elsewhere (e.g., Cooper et al., 2000; Cooper et al., 1995; Cooper et al., 1992) as well as independently predicting multiple types of alcohol and drug use (Cooper et al., 1995).

\section{Distress}

Both anxious and depressive symptomatology are highly related indicators of overall psychological distress (Watson \& Clark, 1992) and, as discussed earlier, are associated with CSA (Jarvis \& Copeland, 1997; Neumann et al., 1996; Weiss et al., 1999; Wilsnack et al., 1997). Reflecting these relationships, a composite distress measure was created from depression and anxiety measures. Depressive symptoms were measured with the 13-item version of the Beck Depression Inventory (BDI; Beck, Ward, Mendelsohn, Mock, \& Erbaugh, 1961). The BDI is one of the most commonly used self-report measures of depressive symptomatology. Anxiety was measured with the Beck Anxiety Index (BAI; Beck, Epstein, Brown, \& Steer, 1988). This 21-item self-report scale taps somatic, affective, and cognitive symptoms associated with anxiety. The time frame for symptoms for both measures was "the past week." Both the BDI and the BAI had good reliabilities (Cronbach's $\alpha \mathrm{s}=.83$ and .89 , respectively). Participants' scores on the BDI and the BAI were standardized and then averaged. Scores on the composite index ranged from -1.09 to +5.20 .

\section{Alcohol Problems}

Drinking problems were assessed with 12 items designed to yield a Diagnostic and Statistical Manual of Mental Disorders, third edition, revised (DSM-III-R; American Psychiatric Association, 1987) diagnosis of 
abuse or dependence, taken from the National Institute of Mental Health Diagnostic Interview Schedule (Robins et al., 1981). Drinking problem items were framed in terms of life history. Representative problems include experiences of increased alcohol tolerance, having the shakes or blackouts, or interference with employment. An index of drinking problems was created by summing the 12 items. Given the severity of these problems, only respondents who reported consuming five or more alcoholic beverages in a single day or a sufficient amount of alcohol to get "drunk or very high" in the past 12 months were asked to respond to the drinking problems items. Respondents who did not answer these questions were coded as having no ("0") alcohol problems. The number of reported problems ranged from 0 to 7 . Substantial evidence has been reported by Robins et al. (1981) to support the validity and reliability of this index as an indicator of clinically significant alcohol-related problems.

\section{Procedure}

Data were collected through in-person interviews by specially trained interviewers. Interviews typically lasted around $90 \mathrm{~min}$ and were usually conducted in participants' homes. The interviews were designed to investigate a number of topics, of which only a few are reported here (for further information, see Nolen-Hoeksema, Larson, \& Grayson, 1999).

\section{Analytic Strategy}

Overall model fit was tested in the context of structural equation modeling (SEM). Path coefficients and model fit were estimated using AMOS Version 4.01 software using the full information maximum likelihood estimator (Arbuckle \& Wothke, 1995-1999). Multiple indicators were used to assess model fit. In the context of SEM, significant goodness-of-fit chi-square statistics indicate lack of a good model; this statistic becomes more sensitive as sample size increases so additional indices are required to assess fit in a large sample. The difference among the chi-square statistics of nested models provides a statistical test of relative fit. Mediation was tested formally following the suggestions by MacKinnon and colleagues (MacKinnon \& Dwyer, 1993; MacKinnon, Warsi, \& Dwyer, 1995). Significance of indirect effects was calculated using Goodman's formula and MacKinnon and Tien's (1999) extensions of Goodman (1960). In addition, the fit indices of models with and without a given direct effect (e.g., the path from CSA to the coping motive) were compared to assess the results of including or dropping direct effects on overall model fit.

Only standardized regression weights and path coefficients are reported. Initial data analyses revealed that the data for distress, drinking to cope, and alcohol problems were positively skewed; as a result, these scales were logarithmically transformed to increase their symmetry to an acceptable level. All reported analyses were performed on the transformed variables.

\section{Results}

\section{Correlates of CSA}

Consistent with our predictions, childhood sexual assault was positively associated with both the coping and enhancement motives. CSA also was positively associated with psychological distress and incidence of alcohol problems. A complete matrix of zero order correlations is shown in Table 1.

In support of the proposition that CSA is associated with increased alcohol-related problems, we found that women with a history of CSA were twice as likely to report any problems related to alcohol consumption (27\%) as women who did not have this history $(13 \%), \chi^{2}(1, N=$ $697)=17.40, p<.001$. They also were 1.5 times more likely to report being drunk in the last year (43\%) than

Table 1. Correlations Among Childhood Sexual Assault, Distress, Drinking to Cope, Drinking to Enhance, and Alcohol Problems

\begin{tabular}{lccccc}
\hline Variable & 1 & 2 & 3 & 4 & 5 \\
\hline 1. Childhood sexual assault & - & $.14^{* * *}$ & $.09^{*}$ & $.13^{* * *}$ & $.15^{* * *}$ \\
2. Distress & & - & $.19^{* * *}$ & $.08^{*}$ & $.12^{* * *}$ \\
3. Drinking to cope & & - & $.64^{* * *}$ & $.46^{* * *}$ \\
4. Drinking to enhance & & & - & $.45^{* * *}$ \\
5. Alcohol problems & & & \\
\hline Note. $N=697$. & & & \\
$* p<.05 . * * p<.01 . * * * p<.001$. & &
\end{tabular}


those without a CSA history $(27 \%), \chi^{2}(1, N=477)=$ $11.48, p<.01$.

\section{Distress Coping Versus Emotion Regulation Model}

Recall that the distress coping model predicted that the relationship between CSA and alcohol problems would be fully mediated by distress and the coping motive. We began by testing whether the distress coping model could adequately describe the data with the direct effect of CSA on alcohol problems included. This model fit the data poorly, goodness-of-fit $\chi^{2}(4, N=697)=$ $38.80, p<.001$, root-mean-square error of approximation $(\mathrm{RMSEA})=.11$, Akaike information criterion $(\mathrm{AIC})$ $=70.80$, Browne-Cudeck criterion $(\mathrm{BCC})=71.08$. As seen in Fig. 1, the path from CSA to alcohol problems remained significant (path coefficient $=.10, p<.01$ ), although reduced in size from its bivariate relationship ( $r=$ $.15, p<.001)$, suggesting distress and the coping motive partially rather than fully mediated this relationship. This issue is discussed in more detail later. These results did not support the distress coping model when the enhance- ment motive is included in the model but its relationship to alcohol problems is not. The distress coping model's fit suffers because the known association between the enhancement motive and alcohol problems is omitted. In a version of the distress model that drops the enhancement motive entirely (not pictured), this model does fit the data well, goodness-of-fit $\chi^{2}(2, N=697)=3.6, p=.17$, $\mathrm{RMSEA}=.034, \mathrm{AIC}=27.56, \mathrm{BCC}=27.74$.

We then turned to the emotion regulation model. The emotion regulation model adds only one prediction to the distress coping model: it predicts CSA's effect on alcohol problems will be partially mediated by the enhancement motive. We began with asking whether the emotion regulation model fit the data, again including the direct effect of CSA on alcohol problems. This model fit the data well, goodness-of-fit $\chi^{2}(3, N=697)=7.22, p=.07$, RM$\mathrm{SEA}=.04, \mathrm{AIC}=41.22, \mathrm{BCC}=41.52$. The emotion regulation model fit significantly better than the distress coping model as indexed by both a significant decrease in the chi-square goodness-of-fit indices, delta $\chi^{2}(1, N=$ $697)=31.83, p<.001$, and a decrease in the parsimonyoriented indices.

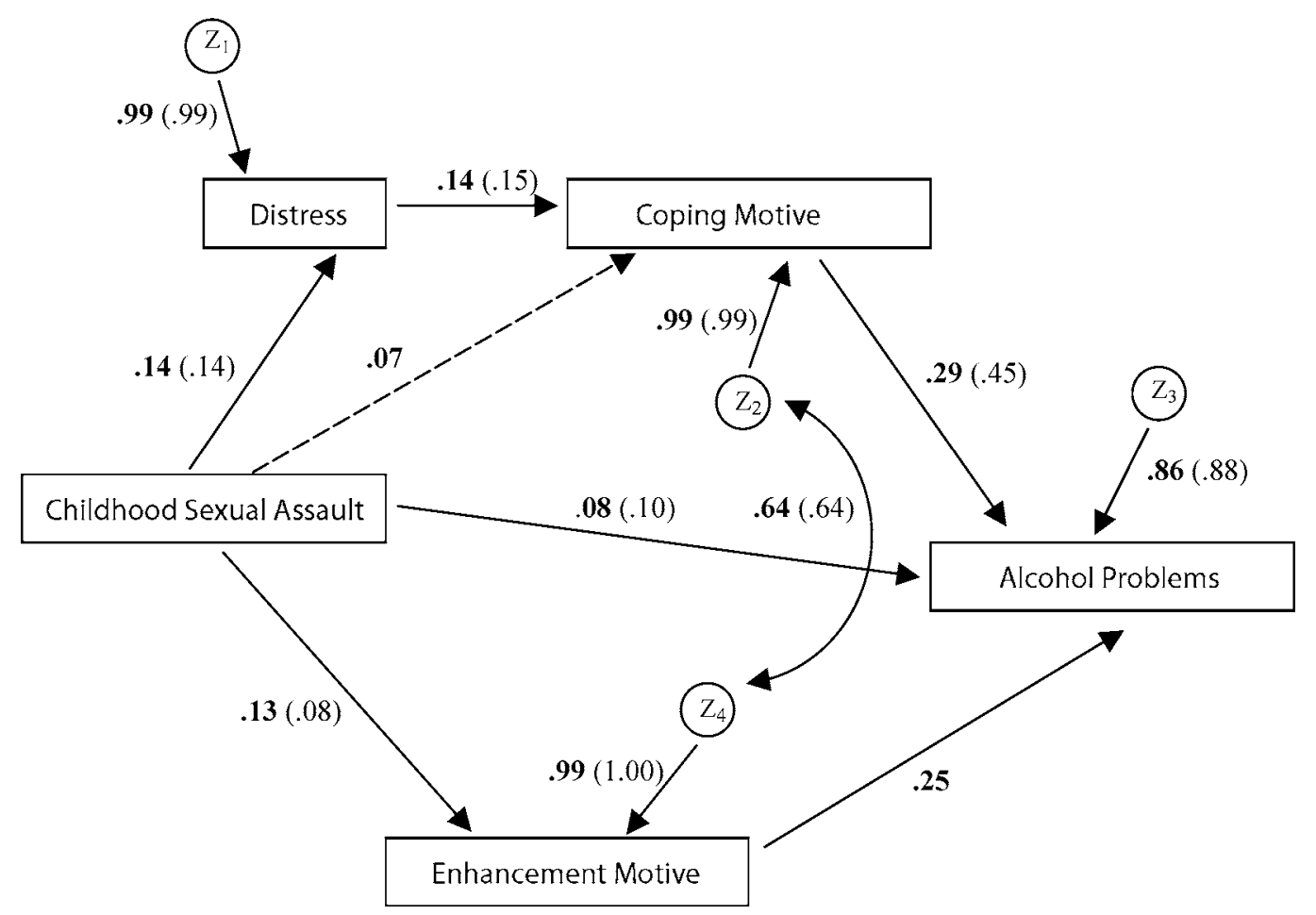

Fig. 1. Distress coping and emotion regulation models. Coefficients in bold are from the emotion regulation model. Coefficients in parentheses are from the distress coping model. All coefficients are standardized. Solid paths are significant at $p<.05$; dashed paths are significant at $p<.10$. The letters $z_{1}$ through $z_{4}$ represent variance in endogenous variables that was unaccounted for by other variables in the model. 
The emotion regulation model has four specific predictions about mediation. It predicts that (a) CSA's relationship to the coping motive is mediated by distress, (b) distress's relationship to alcohol problems is mediated by the coping motive, (c) the two-mediator pathway implied by the first two hypotheses would partially mediate the relationship between CSA and alcohol problems, and (d) the enhancement motive also will partially mediate the relationship between CSA and alcohol problems.

Each prediction was tested separately by adding the relevant direct effect, assessing its statistical significance, its impact on the overall fit indices, and formally testing the significance of the indirect path. To test the first prediction, a path between CSA and the coping motive was added to the model. This path was not quite significant at the $p<.05$ level, path coefficient $=.07, p=.055$. A formal test of the indirect path from CSA through distress to the coping motive, although small, was significant, indirect effect $=.02, z=2.77, p<.01$. There was a trend for the new model to fit better than the original model, delta $\chi^{2}(1, N=697)=3.70, p=.054$, and the parsimonyoriented indices dropped despite the addition of another parameter, goodness-of-fit $\chi^{2}(2, N=697)=3.27, p=$ $.20, \mathrm{RMSEA}=.03, \mathrm{AIC}=39.27, \mathrm{BCC}=39.58$. As a result, the marginally significant path from CSA to the coping motive was retained. Distress partially mediated the effect of CSA on the coping motive.

Recall the second hypothesis predicted that the coping motive would mediate the direct effect of distress on alcohol problems. This prediction was tested by adding a direct path between distress and alcohol problems. As predicted, this path was not significant, path coefficient $=$ $.04, p=.28$, and the resulting model did not fit the data as well as the original emotion regulation model, goodnessof-fit $\chi^{2}(1, N=697)=2.23, p=.14$, RMSEA $=.04$, $\mathrm{AIC}=40.60, \mathrm{BCC}=42.23$; delta $\chi^{2}(1, N=697)=1.04$, $p>.30$. A formal test of the indirect path from distress through the coping motive to alcohol problems was significant, indirect effect $=.04, z=3.68, p<.001$. The second prediction was supported. Returning to the original emotion regulation model, we tested the third prediction that the two-mediator path from CSA through distress and the coping motive would be significant, and it was: indirect effect $=.01, z=2.53, p<.01$.

The fourth prediction was that the enhancement motive also would partially mediate the relationship between CSA and alcohol problems. A direct test of the mediating effect of the enhancement motive on the relationship between CSA and alcohol problems was significant, indirect effect $=.03, z=2.88, p<.01$. Although the path from CSA to alcohol problems was still significant, it had dropped substantially from its bivariate relationship (cor- relation $=.15$, final path coefficient $=.09$ ). The fourth prediction was supported.

\section{Discussion}

Both the enhancement motive and the coping motive partially mediated the relationship between CSA and drinking problems. Previous research has focused on only one of these mechanisms - the desire to cope with negative internal states. The present study proposed a broader framework, the emotion regulation model, that also included the desire to achieve positive emotional states. In general, the coping motive is a better predictor of drinking problems than the enhancement motive, which is reflected in the coping motive's larger direct effect on alcohol problems; however, the mediational path through the enhancement motive (indirect effect $=.02$ ) was the same size as that through distress and the coping motive (indirect effect $=.02$ ). CSA had a roughly equal influence on positive and negative mood regulation. While a sense of happiness is a worthy psychological goal, not all strategies for achieving positive mood states are equally effective. In fact, some of these strategies (e.g., heavy drinking, amphetamine consumption, or risky sex) may have adverse rather than beneficial consequences. Ineffective positive emotion-seeking strategies not only may fail to bring about long-lasting positive affective states but ultimately may lead to an increase in negative emotions. This ironic effect is not only the result of the ineffective positive strategies' failure to work but also indicates the real problems that they may cause. Consistent with the work of Cooper and colleagues (Cooper et al., 1995; Cooper et al., 1992), we found that the motive to achieve a positive emotional state through alcohol consumption could lead to negative consequences such as problems with work and social relationships as well as physical addiction.

This study has several limitations. The correlational study design excludes the possibility of making causal inferences. The variable order may be reversed. It is possible that the proclivity to regulate emotions with alcohol could precede CSA. Alternatively, alcohol consumption has been associated with increased risk of sexual assault for women as well as an adverse outcome of assault (e.g., Kilpatrick, Acierno, Resnick, Saunders, \& Best, 1997).

A number of limitations are the result of the singleitem, retrospective measure of sexual assault. First, retrospective measures may be subject to recall biases. In addition, single-item measures have been associated with underreporting of sexual trauma (Finkelhor \& Brown, 1988); however, Epstein et al. (1998) found a similar 
pattern of results when examining the relationship between childhood rape and alcohol abuse symptoms. Their victimization screening was a series of specific questions that allowed them to assess whether respondents met their definition of childhood rape "as vaginal, anal, or oral penetration" (p. 226). These convergent findings suggest that the pattern of results reported here is not due solely to the phrasing of the question. Unfortunately, detailed analyses of potential moderators such as the perpetrator-survivor relationship and the nature of the assault were not possible. Many CSA survivors are revictimized in adulthood (for a review, see Arata, 2002), and the findings presented here may reflect the cumulative effect of multiple victimizations rather than just CSA alone.

While the sample was relatively large and was recruited using random-digit dialing, certain sample characteristics may limit the generalizability of these results to the population of women who have experienced CSA. Approximately $25 \%$ of those contacted refused to participate in the study, and the final sample had fairly high socioeconomic status (SES). Women with the most debilitating drinking problems may be less likely to be living in situations in which this sampling procedure could reach them (e.g., temporary housing), when contacted be less likely to agree to in-home interviews, and may have lower SES. As a result, women who might have reported the highest number of alcohol problems may have been systematically excluded and the range of our outcome measure truncated. In addition, the sample was stratified on age and, as a result, did not sample women just moving into adulthood (18-25 years old). Alcohol consumption is inversely related to age (e.g., Cooper et al., 1995); it is possible that the most marked effects of CSA on alcohol consumption will be seen in this youngest segment. These factors probably only reduced the strength of our findings, however.

The bivariate relationship between CSA and alcohol problems was low. In their review, Moncreif and Farmer (1998) noted that this relationship is stronger in clinical rather than community samples. This attenuation may be an effect of our community sample. Our outcome measure of alcohol problems reflected severe levels of dysfunction; it is possible that the relationship between CSA and problems is stronger when a broader range of problems are used. Nonetheless, it is remarkable, and potentially clinically important, that women with a history of CSA were twice as likely as women without a history to have alcohol problems and $50 \%$ more likely to have been drunk in the last year.

The relationships between the motives variables and alcohol problems were stronger, although this may be due in part to possible overlap in these constructs and their measures; however, we note that the motives measures and alcohol problems measure were only moderately correlated, indicating the discriminant validity of the two measures. Moreover, we would expect the relationship between the motives and alcohol problems measures to be stronger than the relationship between the measure of CSA and alcohol problems because motives would have a more proximal influence on drinking behavior than would CSA.

The drinking motivations only partially mediated the relationship between childhood sexual assault and alcohol problems, indicating that other psychological processes are at work. Future studies could address other emotion regulation mediators such as rumination and suppression. While the current study focused on only one predictor (CSA) of alcohol-related problems in women, it should be acknowledged that research has identified many other predictors (e.g., family history and antisociality; Zucker, Ellis, Bingham, \& Fitzgerald, 1996).

This study also has a number of strengths. The present study adds to the scarce empirical literature investigating the psychological mechanisms that underlie the connection between CSA and adult problems with alcohol consumption. The sample was drawn from the community at large and drew on the experiences of women throughout the life span. Finally, this article presents a conceptually driven framework that provides specific mechanisms for explaining maladaptive emotion regulation behaviors in women with a history of CSA.

The results of this study suggest that women with a history of CSA are vulnerable to ineffective emotion regulation strategies. This vulnerability is particularly troubling given their likelihood of experiencing psychological distress and difficulty in achieving positive emotional states. Frederickson (2000) suggested that effective strategies such as those used in relaxation therapies may increase positive emotions and ultimately may lead to greater personal coping resources. Clinical interventions that help clients develop these adaptive strategies may be particularly effective in alleviating psychological distress and increasing positive emotional states as well as preventing the negative outcomes associated with maladaptive strategies such as heavy drinking.

\section{Acknowledgments}

This research was supported by Public Health Grant MH 51817. We are indebted to Laura Klem for statistical advice. We thank Jennifer Waltz and Katherine Rosenblum for commenting on an earlier version of this article. 


\section{References}

American Psychiatric Association. (1987). Diagnostic and statistical manual of mental disorders (3rd ed., Rev.). Washington, DC: Author.

Arata, C.M. (2002). Child sexual abuse and sexual revictimization. Clinical Psychology: Science \& Practice, 9, 135-164.

Arbuckle, J.L. (2001). Amos 4.01 [Computer software]. Chicago: Smallwaters.

Arbuckle, J.L., \& Wothke, W. (1995-1999). AMOS 4.0 user's guide. Chicago: SPSS, Inc.

Beck, A.T., Epstein, N., Brown, G., \& Steer, R.A. (1988). An inventory for measuring anxiety: Psychometric properties. Journal of Consulting and Clinical Psychology, 56, 893-897.

Beck, A.T., Ward, C.H., Mendelsohn, M., Mock, J., \& Erbaugh, J. (1961). An inventory for measuring depression. Archives of General Psychiatry, 4, 561-571.

Burnam, M.A., Stein, J.A., Golding, J.M., Siegel, J.M., Sorenson, S.B., Forsythe, A.B., et al. (1988). Sexual assault and mental disorders in a community population. Journal of Consulting and Clinical Psychology, 56, 843-850.

Conger, J.J. (1956). Alcoholism: Theory, problem, and challenge: II. Reinforcement theory and the dynamics of alcoholism. Quarterly Journal of Studies on Alcohol, 17, 296-305.

Cooper, M.L., Agocha, V.B., \& Sheldon, M.S. (2000). A motivational perspective on risky behaviors: The role of personality and affect regulatory processes. Journal of Personality, 68, 10591088.

Cooper, M.L., Frone, M.R., Russell, M., \& Mudar, P. (1995). Drinking to regulate positive and negative emotions: A motivational model of alcohol use. Journal of Personality and Social Psychology, 69, 990-1005.

Cooper, M.L., Russell, M., Skinner, J.B., \& Windle, M. (1992). Development and validation of a three-dimensional measure of drinking motives. Psychological Assessment, 4, 123-132.

Davis, J.L., \& Petretic-Jackson, P.A. (2000). The impact of child sexual abuse on adult interpersonal functioning: A review and synthesis of the empirical literature. Aggression \& Violent Behavior, 5, 291328.

Diener, E., \& Emmons, R.A. (1984). The independence of positive and negative affect. Journal of Personality \& Social Psychology, 47, 1105-1117.

Epstein, J.N., Saunders, B.E., Kilpatrick, D.G., \& Resnick, H.S. (1998). PTSD as a mediator between childhood rape and alcohol use in adult women. Child Abuse \& Neglect, 22, 223234.

Finklehor, D., \& Brown, A. (1988). Assessing the long-term impact of child sexual abuse: A review and conceptualization. In L.E.A. Walker (Ed.), Handbook on sexual abuse of children: Assessment and treatment issues (pp. 55-71). New York: Springer.

Finkelhor, D., Hotaling, G., Lewis, I.A., \& Smith, C. (1990). Sexual abuse in a national survey of adult men and women: Prevalence, characteristics, and risk factors. Child Abuse \& Neglect, 14, 1928

Frederickson, B.L. (2000). Cultivating positive emotions to optimize health and well-being. Prevention \& Treatment, 3, n.p.

Goodman, L.A. (1960). On the exact variance of products. Journal of the American Statistical Association, 55, 708-713.

Gray, J.P. (1990). Brain systems that mediate both emotion and cognition. Cognition and Emotion, 4, 269-288.

Harrison, P.A., Fulkerson, J.A., \& Beebe, T.J. (1997). Multiple substance use among adolescent physical and sexual abuse victims. Child Abuse \& Neglect, 21, 529-539.

Hayes, S.C. (1987). A contextual approach to therapeutic change. In N.S. Jacobson (Ed.), Psychotherapists in clinical practice: Cognitive and behavioral perspectives (pp. 327-387). New York: Guilford Press.
Jarvis, T.J., \& Copeland, J. (1997). Child sexual abuse as a predictor of psychiatric co-morbidity and its implications for drug and alcohol treatment. Drug and Alcohol Dependence, 49, 61-69.

Jarvis, T.J., Copeland, J., \& Walton, L. (1998). Exploring the nature of the relationship between child sexual abuse and substance use among women. Addiction, 93, 865-875.

Kilpatrick, D.G., Acierno, R., Resnick, H.S., Saunders, B.E., \& Best, C.L. (1997). A two-year longitudinal analysis of the relationships between violent assault and substance use in women. Journal of Consulting and Clinical Psychology, 65, 834-847.

Langeland, W., \& Hartgers, C. (1998). Child sexual and physical abuse and alcoholism: A review. Journal of Studies on Alcohol, 59, 336349.

MacKinnon, D.P., \& Dwyer, J.H. (1993). Estimating mediated effects in prevention studies. Evaluation Review, 17, 144-158.

MacKinnon, D.P., \& Tien, J.Y. (1999). A simulation study of multiple path mediation. Unpublished manuscript, Arizona State University, Tempe.

MacKinnon, D.P., Warsi, G., \& Dwyer, J.H. (1995). A simulation study of mediated effect measures. Multivariate Behavioral Research, 30, $41-62$.

McCloskey, L.A. (1997). The continuum of harm: Girls and women at risk for sexual abuse across the lifespan. In D. Cicchetti \& S. Toth (Eds.), Rochester Symposium on Developmental Psychopathology, Vol. 8. Developmental perspectives on trauma: Theory, research, and intervention (pp. 553-578). Rochester, NY: University of Rochester Press.

Moncrieff, J., \& Farmer, R. (1998). Sexual abuse and the subsequent development of alcohol problems. Alcohol and Alcoholism, 33, 592-601

Neumann, D.A., Houskamp, B.M., Pollock, V.E., \& Briere, J. (1996). The long-term sequelae of childhood sexual abuse in women: A meta-analytic review. Child Maltreatment: Journal of the American Professional Society on the Abuse of Children, 1, 6-16.

Nishith, P., Resick, P.A., \& Mueser, K.T. (2001). Sleep difficulties and alcohol use motives in female rape victims with posttraumatic stress disorder. Journal of Traumatic Stress, 14, 469-479.

Nolen-Hoeksema, S., Larson, J., \& Grayson, C.E. (1999). Explaining the gender difference in depressive symptoms. Journal of Personality and Social Psychology, 77, 1061-1072.

Pohorecky, L.A. (1991). Stress and alcohol interaction: An update on human research. Alcoholism: Clinical and Experimental Research, $15,438-459$

Polusny, M.A., \& Follette, V.M. (1995). Long-term correlates of child sexual abuse: Theory and review of the empirical literature. Applied \& Preventive Psychology, 4, 143-166.

Robins, L.E., Helzer, J.E., \& Croughan, J.L. (1981). National Institute of Mental Health diagnostic interview: Its history, characteristics, and validity. Archives of General Psychiatry, 38, 383-389.

Romans, S.E., Gendall, K.A., Martin, J.L., \& Mullen, P.E. (2001). Child sexual abuse and later disordered eating: A New Zealand epidemiological study. Journal of Eating Disorders Special Issue, 29, 380392.

Saunders, B.E., Villeponteaux, L.A., Lipovsky, J.A., Kilpatrick, D.G., \& Veronen, L. J. (1992). Child sexual assault as a risk factor for mental disorders among women: A community survey. Journal of Interpersonal Violence, 7, 189-204.

Vaillant, G.E. (2000). Adaptive mental mechanisms: Their role in a positive psychology. American Psychologist, 55, 89-98.

Watson, D., \& Clark, L.A. (1992). Affects separable and inseparable: On the hierarchical arrangement of the negative affects. Journal of Personality and Social Psychology, 62, 489-505.

Weiss, E.L., Longhurst, J.G., \& Mazure, C.M. (1999). Childhood sexual abuse as a risk factor for depression in women: Psychosocial and neurobiological correlates. American Journal of Psychiatry, 156, 816-828.

Wilsnack, S.C., Vogeltanz, N.D., Klassen, A.D., \& Harris, T.R. (1997). Childhood sexual abuse and women's substance abuse: National survey findings. Journal of Studies on Alcohol, 58, 264-272. 
Wyatt, G.E. (1988). The relationship between childhood sexual abuse and adolescent sexual function in Afro-American and White American woman. Annals of New York Academy of Sciences, $528,111-122$.

Zierler, S., Feingold, L., Laufer, D., Velentgas, P., Knatrowitz-Gordon, I., \& Mayer, K. (1991). Adult survivors of childhood sexual abuse and subsequent risk for HIV infection. American Journal of Public Health, 81, 572-575.

Zucker, R.A., Ellis, D.A., Bingham, C.R., \& Fitzgerald, H.E. (1996). The development of alcoholic subtypes: Risk variation among alcoholic families during the early childhood years. Alcohol Health and Research World, 20, 46-55. 